\title{
Habilidades Maternas de Mulheres Vítimas de Violência Doméstica: Uma Revisão da Literatura
}

Maternal Skills of Women Victims of Domestic Violence:
Literature Review

Habilidades Maternas de Mujeres Víctimas de Violencia

Doméstica: Una Revisión de la Literatura

Sabrina Mazo D’Affonseca \& Lúcia Cavalcanti de

Albuquerque Williams

Universidade

Federal de São Carlos

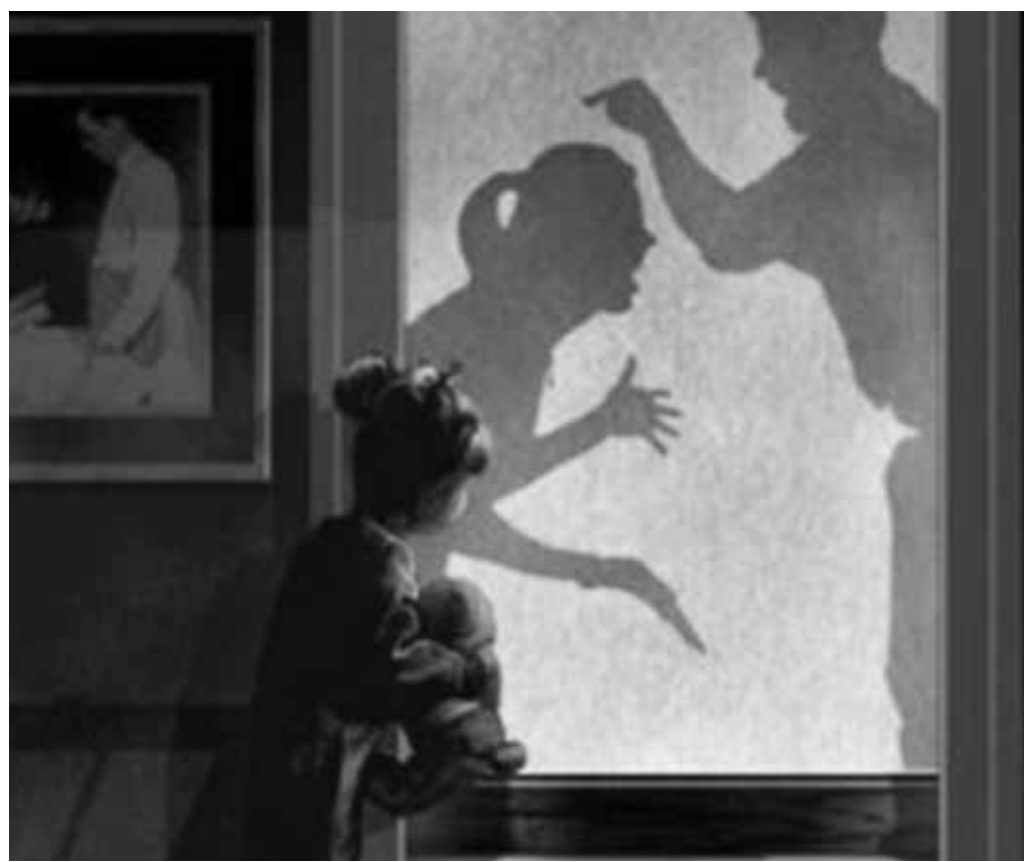


Resumo: O presente estudo objetivou revisar a literatura nacional e internacional de 2007 a 2010 sobre violência doméstica e habilidades maternas nas seguintes bases de dados: Bireme, Web of Science, Lilacs e Medline. A busca resultou em 85 artigos, sendo analisados 28 que se relacionavam com a proposta deste estudo, todos internacionais, divididos em quatro categorias: (1) prevalência, (2) intervenção, (3) relação entre violência conjugal e habilidades maternas e (4) impacto da violência conjugal nos filhos. A análise dos artigos permitiu verificar que uma parcela expressiva de crianças presencia desentendimentos violentos entre os pais e são afetadas direta ou indiretamente por eles. Nos artigos em que se buscou avaliar a relação entre a violência conjugal e as habilidades maternas, os resultados demonstraram os impactos negativos da exposição à violência conjugal e a importância de um relacionamento mãe-filho positivo para a diminuição dos problemas de comportamento dos filhos, sendo esse um fator importante quando se pensa em intervir nessa população. Destaca-se que todos os dados foram obtidos por meio de autorrelato das mulheres, sendo sugeridas pesquisas futuras que utilizem outras formas de coleta de dados, como fontes de informação diversas ou estudos observacionais sistematizados.

Palavras-chave: Violência na família. Abuso da criança. Conflito conjugal. Relações mãe-criança. Mães.

Abstract: This study reviewed the national and the international literature of domestic violence regarding maternal skills from 2007 to 2010.The following data base were used: Bireme, Web of Science, Lilacs and Medline. Out of the 85 abstracts, 28 were related to the topic of this paper (all international) and they were divided into four categories: (1) prevalence, (2) intervention, (3) the relationship between domestic violence and maternal skills and (4) the impact of marital violence on children. Analysis of the studies showed that an expressive number of children witnessed violent conflicts between their parents, and that they were directly or indirectly affected by these conflicts. In studies which sought to evaluate the relationship between domestic violence and maternal skills the results showed the negative impact of exposure to marital violence and the importance of a positive mother-child relationship for the reduction of behavior problems in children, an important variable when considering the intervention with this population. It is noteworthy that all data were collected by self-report measures, therefore it is suggested that future research uses other types of data collection as different sources of information or systematic observational studies.

Keywords: Family violence. Child abuse. Marital conflict. Mothering. Mother.

Resumen : El presente estudio objetivó revisar la literatura nacional e internacional de 2007 a 2010 sobre violencia doméstica y habilidades maternas en las siguientes bases de datos: Bireme, Web of Science, Lilacs y Medline. La búsqueda resultó en 85 artículos, siendo analizados 28 que se relacionaban con la propuesta de este estudio, todos internacionales, divididos en cuatro categorías: (1) prevalencia, (2) intervención, (3) relación entre violencia conyugal y habilidades maternas e (4) impacto de la violencia conyugal en los hijos. El análisis de los artículos permitió verificar que una parte expresiva de niños está presente durante desentendimientos violentos entre los padres y son afectados directa o indirectamente por ellos. En los artículos en los que se buscó evaluar la relación entre la violencia conyugal y las habilidades maternas, los resultados demostraron los impactos negativos de la exposición a la violencia conyugal y la importancia de una relación madre e hijo positiva para la disminución de los problemas de comportamiento de los hijos, siendo ése un factor importante cuando se piensa en intervenir en esa población. Es importante mencionar que todos los datos fueron obtenidos a través de auto relato de las mujeres, siendo presentadas como sugerencias investigaciones futuras que utilicen otras formas de colecta de datos, como fuentes de información variadas o estudios de observación sistematizados.

Palabras clave: Violencia domestica. Abuso de niños. Conflicto marital. Relaciones madre-niño. Habilidades maternas. Madres.

A violência é um problema social grave que produz várias vítimas. $\mathrm{O}$ fenômeno da violência contra a mulher não é recente, entretanto, nas últimas décadas, em especial com o movimento feminista, as atenções dos profissionais de diferentes áreas de atuação têm se voltado para essa questão.

A violência contra a mulher foi reconhecida, pelas Nações Unidas (ONU), em 1993, como um obstáculo ao desenvolvimento, à paz e aos ideais de igualdade entre os seres humanos, sendo essa considerada uma violação aos direitos humanos (Hermann \& Barsted, 2000), ou seja, a violência contra a mulher é um problema social e de saúde pública que consiste em um fenômeno mundial e que não respeita fronteiras de classe social, raça/ 
etnia, religião, idade e grau de escolaridade. Os episódios comumente ocorrem no âmbito familiar, no espaço privado, e os agressores, em geral, são homens, na maioria das vezes seus parceiros (Arias \& Ikeda, 2006; Lutzker, 2006; Vasconcelos, 2002).

Apesar da dificuldade em se determinar precisamente os casos de violência contra a mulher, dados de diferentes pesquisas os têm apontado como um dos delitos mais frequentes no mundo, responsável por sequelas nocivas ao seu desenvolvimento (Williams, 2001). Dentre os sintomas apresentados pelas mulheres vítimas de violência, destacam-se: dor crônica, visita frequente ao médico, uso/ abuso de medicamentos, uso/abuso de álcool, história de pensamentos/tentativas de suicídio, depressão moderada ou grave, suspeita de maus-tratos infantis como mãe, problemas para dormir (insônia, pesadelos), agitação, ansiedade ou nervosismo, pensamento confuso, dificuldade de tomar decisões, distúrbios sociais, ausência de contato visual e visão rígida dos papéis de homem/mulher (Adeodato, Siqueira, \& Souza, 2005; Williams, 2001; Sinclair, 1985).

Além disso, cabe destacar que vários pesquisadores consideram a violência contra o parceiro como um fator de risco para a violência contra a criança (Capaldi, Kim, \& Pears, 2009; Williams, Padovani, \& Brino, 2009; O'Leary \& Woodin, 2006; Appel \& Holden, 1998), e pesquisas citadas por Capaldi, Kim e Pears (2009) que buscam relacionar esses dois fenômenos (violência contra o parceiro e violência contra a criança) indicam que crianças em cuja família ocorre violência contra o parceiro tem uma probabilidade de duas a quatro vezes maior de serem vítimas de maus-tratos, quando comparadas com crianças cujas famílias não vivenciam esse fenômeno.
Slep e O'Leary (2001) destacam alguns fatores de risco comuns para a violência contra o parceiro e a violência contra crianças em diferentes níveis: contextual (pobreza e estresse), relacionamentos sociais (agressão na família de origem, parentagem pobre na família de origem, discórdia entre os pais, violência entre os pais e pouca satisfação com o relacionamento) e pessoais (impulsividade, agressividade, baixa resolução de problemas, depressão, abuso de substância, história de comportamento antissocial - problemas de conduta e delinquência).

Considerando a situação vivenciada pela mulher vítima de violência, é importante analisar os impactos que essa situação pode acarretar para os seus filhos. Ainda que eles não sejam vítimas diretas da violência, estão presentes em um ambiente hostil, pouco saudável para o seu desenvolvimento.

Além disso, pode-se supor que a mãe que vivencia agressões físicas e psicológicas do parceiro muitas vezes não está em condições de estabelecer um relacionamento adequado com os filhos. As pesquisas a respeito dos efeitos da violência doméstica nos comportamentos da mãe em relação aos filhos, aqui considerado como maternagem, indicam que, devido a todas as consequências que a violência pode acarretar na saúde física e psicológica dessa mulher, elas apresentariam maior dificuldade nos cuidados com os filhos do que as mães que não tivessem esse histórico. De fato, estudos têm demonstrado que a qualidade da maternagem e a habilidade de ambos os pais em lidar com as necessidades da criança são comprometidas em ambientes domésticos violentos (Holt, Buckley \& Whelan, 2008). Alguns autores chegam a relacionar a violência sofrida pela mulher como um fator de risco para essa mulher vir a agredir os filhos (Capaldi, Kim, 
Alguns autores chegam a relacionar a violência sofrida pela mulher como um fator de risco para essa mulher vir a agredir os filhos (Capaldi, Kim, \& Pears, 2009; Holden, 2007; Chaffin \& Schmidt, 2006; Dahlberg \& Simon, 2006; O'Leary \&

Woodin, 2006), sendo que, em pesquisa realizada por Casanueva, Martin e Runyan (2009), contatou-

se que as mães submetidas à violência doméstica

tinham um risco maior de vir a perpetrar maustratos quando comparadas com mães que não apresentaram esse histórico.
\& Pears, 2009; Holden, 2007; Chaffin \& Schmidt, 2006; Dahlberg \& Simon, 2006; O'Leary \& Woodin, 2006), sendo que, em pesquisa realizada por Casanueva, Martin e Runyan (2009), contatou-se que as mães submetidas à violência doméstica tinham um risco maior de vir a perpetrar maus-tratos quando comparadas com mães que não apresentaram esse histórico.

Williams e Araújo (2009) realizaram uma revisão dos aspectos teóricos envolvidos no ensino de habilidades maternas a mulheres vítimas de violência conjugal, de acordo com a literatura da área, e não encontraram projetos de intervenção voltados para o ensino de habilidades maternas a mulheres com tal histórico. As autoras, porém, encontraram artigos que fornecem pistas sobre quais seriam as habilidades maternas de mulheres que sofrem a violência do parceiro.

Assim, o presente estudo visa revisar a literatura da área de 2007 a 2010 sobre violência doméstica e habilidades maternas. Para tanto foram pesquisadas as mesmas bases de dados utilizadas por Williams e Araújo (2009) - Bireme, Web of Science, Lilacs e Medline - com as seguintes palavras-chave: estilo parental AND violência doméstica; violência doméstica AND paternagem/ maternagem; intervenção familiar AND problemas de comportamento infantil AND mulheres agredidas; parental style AND domestic violence; domestic violence AND parenting/mothering; family intervention AND child behavior problems AND abused women.

Foram encontrados 85 resumos de artigos, e apenas um deles com as palavras-chave em português. Desse total, foram excluídos 57 repetidos, ou seja, quando o mesmo artigo aparecia em mais de uma base de dados, os que não apresentavam informações suficientes, como, por exemplo, qual o método empregado, o número de participantes etc., ou aqueles que consistiam em artigos teóricos ou eram artigos que descreviam estudos sobre punição física, sobre fatores de risco aos maus-tratos infantis, que avaliavam apenas o papel paterno ou que eram muito abrangentes e não se enquadravam em uma categoria específica (Mele, 2009; Tischler, Rademeyer, \& Vostanis, 2007; Constantino, Crane, Noll, Doswell, \& Braxter, 2007; Edelson, Hokoda, \& Ramos-Lira, 2007). Após uma análise do conteúdo dos 28 artigos restantes, estes foram divididos em quatro categorias, levando-se em conta os achados principais da revisão: (1) prevalência da exposição à violência conjugal, (2) intervenção em mães, crianças e famílias expostas à violência conjugal, (3) consequências da violência conjugal para as habilidades maternas e (4) impacto da violência conjugal nos filhos. Metade dos artigos (50\%) encontrados descreve os efeitos da violência conjugal nas habilidades maternas, 25\%, o impacto da violência conjugal nos filhos, $14,3 \%$ descrevem intervenções realizadas com crianças, mulheres e famílias expostas à violência conjugal e 10\% dos estudos estimam a prevalência da exposição das crianças à violência conjugal.

\section{Prevalência de crianças expostas à violência conjugal}

Nessa categoria, encontram-se dois estudos que buscaram estimar a prevalência de desentendimentos violentos relatados que ocorreram em lares de crianças norteamericanas (Moore, Probst, Tompkins, Cuffe, \& Martin, 2007; Probst, Wang, Martin, Moore, Paul, \& Samuels, 2008) e um terceiro estudo que procurou estimar a prevalência e a natureza da exposição e o envolvimento da criança em delitos de violência doméstica investigados pelo Sistema Judiciário dos EUA (Fusco \& Fantuzzo,2009). 
Tanto Moore et al. (2007) quanto Probst et al. (2008) utilizaram os dados coletados em 2003 pela National Survey of Children's Health (Pesquisa Nacional sobre a Saúde da Criança), sendo que os últimos fizeram um recorte com famílias nativas do Alasca (NA) e famílias indígenas norte-americanas (IA) que tinham filhos de alguns Estados norteamericanos (Alaska, Arizona, Montana, Novo México, Dakota do Norte, Oklahoma e Dakota do Sul ).

Moore et al. (2007) contaram com uma amostra de 99.660 pessoas. Os pesquisadores classificaram os desentendimentos com base em como a família lidava com desacordos sérios, podendo os mesmos ser violentos (o desentendimento envolvia bater ou jogar coisas, mesmo que raramente) ou calorosos (gritos e discussões calorosas). Os resultados obtidos indicaram que, no geral, $10,3 \%$ das crianças viviam em lares com desacordos violentos, sendo os mesmos mais prevalentes em lares negros $(15,1 \%)$, hispânicos (11,3\%) e brancos (8,6\%). As áreas urbanas tiveram a maior prevalência $(10,7 \%)$ quando comparadas com grandes localidades rurais (8,3\%-9,9\%). Assim, os pesquisadores concluíram que os pais que vivem em localidades rurais tinham uma probabilidade menor de relatar desentendimentos violentos quando comparados com aqueles que viviam em ambiente urbano. Crianças negras tinham maior probabilidade de estarem expostas, tanto a desentendimentos violentos quanto calorosos, do que crianças brancas. Pais que relataram maior estresse parental tinham maior porcentagem de desentendimentos violentos e calorosos quando comparados com pais que relataram menor estressse parental. Portanto, embora fatores demográficos e culturais possam influenciar o estilo do desacordo, os autores consideram que o estresse parental seja instrumental para o desenvolvimento de desentendimentos violentos, sendo que pais com dificuldades com a parentagem seriam considerados um grupo de alto risco.

Nesse mesmo sentido, os resultados de Probst et al. (2008) indicaram que desentendimentos violentos foram relatados por $8,4 \%$ de IA/ NA e por $8,4 \%$ de famílias brancas. A probabilidade de desentendimentos violentos era marcadamente maior entre os pais que relaram mais estresse parental, tanto para famílias IA/NA quanto para famílias brancas. Estresse parental alto tinha efeitos similares nas estatísticas para discussão calorosa. Além disso, ter um filho com necessidades especiais foi associado a estresse parental na amostra pesquisada.

Assim, os estudos citados acima demonstram que as dificuldades encontradas pelos pais na educação dos filhos podem ser um fator de risco para o desentendimento do casal e, em consequência, para a violência conjugal, indicando que seria importante a existência de serviços de apoio e de aconselhamento psicológico voltados para acolher os pais que estiverem encontrando dificuldade no manejo do comportamento dos filhos. Os mesmos poderiam ser implementados, de forma universal, em serviços públicos de saúde (unidade básica de saúde, etc.) e em setores da educação (escolas públicas, EMEIS, CEMEIS, etc).

Já Fusco e Fantuzzo (2009) utilizaram um modelo de desenvolvimento epidemiológico para explorar a prevalência e a natureza da exposição e envolvimento da criança em delitos de violência doméstica investigados pelo sistema Judiciário. No ano estudado, 1581 delitos de violência contra a mulher foram investigados, e, em 43\% deles, havia uma criança na casa e quase todas essas 
crianças (95\%) experenciaram exposição sensorial à violência. $\mathrm{O}$ modelo de regressão logística revelou relação entre a exposição da criança e características do evento com o ferimento da vítima, agressão mútua e prisão do agressor. A pesquisa também investigou os tipos de envolvimento da criança nos eventos de violência, sendo identificados três tipos distintos: (1) a criança era parte do evento precipitador, (2) a criança pediu socorro e (3) a criança foi fisicamente envolvida. Os resultados destacam a importância de se desenvolver um sistema de vigilância global para assegurar que a criança exposta à violência doméstica seja vista e encaminhada a serviços apropriados.

Vale destacar que não foi encontrado qualquer estudo nacional que vise a determinar a prevalência de crianças expostas à violência conjugal e os tipos de exposição mais frequentes na nossa população, devendo esses aspectos relevantes serem analisados para que haja implementação de serviços e intervenções eficazes para essa população.

\section{Intervenção em mães, crianças e famílias expostas à violência conjugal}

No total, foram encontrados quatro estudos que descreviam algum tipo de intervenção realizada com diferentes populações: mulheres vítimas de violência doméstica (Holigrocki, Crain, Bohr, Young, \& Bensman, 2009), crianças expostas à violência conjugal (Jouriles, McDonald, Rosenfield, Stephens, Corbitt-Shindler, \& Miller, 2009), famílias notificadas ao programa de proteção a crianças dos Estados Unidos (Casanueva, Martin, Runyan, Barth, \& Bradley, 2008) e famílias consideradas de alto-risco (Duggan, Berlin, Cassidy, Burrell, \& Tandon, 2009).
Um dos estudos, de Holigrocki et al. (2009), descreveu a avaliação e o tratamento de mãe vítima de violência conjugal e o filho de 10 anos de idade. Os dois estavam abrigados no momento em que ocorreu a intervenção. Os autores utilizaram a Avaliação da Interação Pai-Criança II - Modificação das Atribuições dos Pais (Parent-Child Interaction AssessmentII Modifying Attributions of Parents) (PCIA-II/ MAP; Bohr, 2005), tratamento estruturado, de caráter breve, que utiliza gravações de vídeo de uma situação de jogo entre a mãe e o filho. O jogo envolve a utilização de brinquedos em forma de animais e pessoas, e os jogadores devem completar uma história relacionada a uma viagem ao zoológico. Após as gravações, o terapeuta mostra alguns trechos do vídeo para a mãe, tecendo comentários e reflexões a respeito da interação, para modificar a maneira como a mãe interpreta os comportamentos do filho. A avaliação inicial da mãe demonstrou que a mesma estava depressiva, amedrontada e muito estressada, com um estilo parental severo. O garoto experenciava estresse significativo, tinha problemas de comportamento e via os adultos como duros, frágeis, irresponsáveis e indisponíveis. Após a intervenção, os dados obtidos indicaram menor depressão e um aumento na sensibilidade parental, contudo, o estresse parental e os comportamentosproblema do filho permaneceram elevados. Tal descrição da intervenção realizada fornece pistas de estratégias e materiais a serem empregados com essa população, destacando-se o uso do vídeo-feedback. Entretanto, seria importante realizar estudos com uma população maior para se obter dados mais consistentes.

Nesse sentido, os dados de Jouriles, McDonald, Rosenfield, Stephens, CorbittShindler e Miller (2009) são mais consistentes, visto que realizaram um estudo com tentativas 
Já Duggan et al. (2009) verificaram se a depressão materna e o apego inseguro eram moderadores dos impactos de um serviço domiciliar para famílias no Alasca destinado a mães de alto risco e seus filhos. Trezentas e vinte e cinco famílias de alto risco foram

randomicamente atribuídas para visita domiciliar $(n=162)$ e serviços comunitários ( $n=163)$, e a depressão materna e o apego inseguro foram avaliados no início da intervenção. clínicas randomizadas do Project Support (Projeto Apoio), comparando os resultados com um grupo-controle. Participaram do estudo 66 famílias recrutadas em uma Casa Abrigo (mãe e filhos) com pelo menos uma criança exibindo níveis clínicos de problemas de conduta. A intervenção envolvia: (a) ensinar a mães habilidades de manejo da criança e (b) promover suporte emocional e instrumental às mães. As famílias eram randomicamente inscritas no Project Support, ou em um serviço similar existente. As mães foram avaliadas em seis ocasiões durante 20 meses, após sua saída da Casa Abrigo. Os resultados indicaram maior redução nos problemas de comportamento das crianças cujas mães participaram do Project Support quando comparadas com o outro grupo de crianças, e as mães que participaram do Project Support exibiram maior redução de comportamentos parentais inconsistentes e punitivos e de sintomas psiquiátricos, quando comparadas com o outro grupo de mães. As mudanças na maternagem e nos sintomas psiquiátricos foram proporcionais aos efeitos dos problemas de conduta das crianças no final do tratamento.

No Brasil, o LAPREV (Laboratório de Análise e Prevenção da Violência), vinculado ao Departamento de Psicologia da Universidade Federal de São Carlos, desenvolve, desde 2008, o Projeto Parceria, que consiste em um programa de intervenção às mães vítimas de violência conjugal, de modo a prevenir problemas de comportamento de seus filhos (Williams, Araújo, D'Affonseca, Maldonado, Patrian, \& Miranda, 2010; Rios, D'Affonseca, Santini, Lessa, Patrian, Miranda, \& Williams, 2010). Uma avaliação dos dados encontrados na pré-intervenção do projeto indica que todas as mães apresentavam déficits tanto nas suas práticas educativas quanto nos aspectos de relacionamento e de comportamento.
Dados pós-intervenção e follow up indicam que houve melhoria nas práticas educativas e na resolução de problemas, bem como redução no nível de depressão e no potencial de risco para abuso.

Casanueva et al. (2008) realizaram um estudo com amostra nacionalmente representativa de famílias investigadas pelo Conselho Tutelar para verificar se o treinamento parental estava relacionado a mudanças nas práticas parentais 18 meses após o treinamento. As mães que receberam o treinamento eram comparadas com mães que não o receberam, mesmo que essas tivessem necessidade similar pelo serviço. Os resultados encontrados demonstraram benefícios modestos na responsividade materna e nos escores parentais para mães de crianças na faixa etária de 3 a 5 anos de idade para as mães que receberam treinamento parental, quando comparadas com mães que não receberam tal treinamento. Entretanto, esses achados não puderam ser confirmados por uma análise multivariada.

Já Duggan et al. (2009) verificaram se a depressão materna e o apego inseguro eram moderadores dos impactos de um serviço domiciliar para famílias no Alasca destinado a mães de alto risco e seus filhos. Trezentas e vinte e cinco famílias de alto risco foram randomicamente atribuídas para visita domiciliar ( $n=162)$ e serviços comunitários ( $n=163$ ), e a depressão materna e o apego inseguro foram avaliados no início da intervenção. Os dados da maternagem e da situação psicossocial da mãe foram mensurados quando as crianças tinham 2 anos de idade por meio de uma entrevista com a mãe, da observação e da revisão de queixas de maus-tratos infantis. A depressão materna e o apego inseguro interagiram como moderadores dos impactos do programa. 
A visita domiciliar teve um impacto maior para mães não depressivas com desconforto moderado a alto em confiança/dependência e para mães depressivas com baixo desconforto confiança/dependência.

É interessante notar que as intervenções descritas acima utilizaram como medidas de eficácia de intervenção dados da saúde mental das mães (depressão), de problemas de comportamento dos filhos e da qualidade da interação mãe-filho. Embora diferentes metodologias tenham sido empregadas, aparentemente, os resultados encontrados foram promissores, indicando que intervir nas mães é uma alternativa viável e eficaz para minimizar os problemas de comportamento dos filhos.

\section{Consequências da violência conjugal para as habilidades maternas}

A maioria dos estudos ( $50 \%, \mathrm{n}=14)$ analisou os impactos da violência conjugal para as habilidades maternas das mulheres vitimizadas $(n=7)$ e o seu impacto nos filhos $(n=7)$, demonstrando a importância que os pesquisadores têm dado a essas questões. Nos estudos a respeito das habilidades maternas das mulheres vitimizadas, observouse uma predominância para a descrição de comportamentos negativos apresentados por essa população (Damant, Lapierre, Lebosse, Thibault, Lessard, Hamelin-Brabant, Lavergne, \& Fortin, 2010; Renner, 2009; Taylor, Guterman, Lee, \& Rathouz, 2009; Kelleher, Hazen, Coben, Wang, McGeehan, Kohl, \& Gardner, 2008).

O estudo de Kelleher et al. (2008) examinou a associação entre agressão física sofrida pela mulher (tanto recente quanto ocorrida há mais de um ano) e as práticas disciplinares relatadas por mulheres (mães ou cuidadores) em amostra nacional de famílias encaminhadas à rede de proteção à criança. Mais de 3.000 cuidadoras participaram da Pesquisa Nacional Sobre o Bem-Estar da Criança e do Adolescente (National Survey of Child and Adolescent Wellbeing - NSCAW), as quais responderam versões diferentes da Escala de Tática de Conflito (Straus, Hamby, Boney-McCoy, \& Sugarman, 1996) para avaliar a violência conjugal e as práticas disciplinares da criança. Os dados obtidos indicaram que $43 \%$ das participantes relataram ter sofrido violência conjugal no ano anterior, sendo que 1.161 relataram ter sofrido violência conjugal, mas não nos últimos 12 meses. Qualquer tipo de exposição à violência conjugal foi associado a aumento nas taxas de agressão psicológica, agressão física e disciplina negligente à criança, quando comparadas a mulheres que não sofreram violência doméstica. Depois de controlar as variáveis específicas (comportamento da criança, fatores demográficos e características maternas), as mulheres vitimizadas, no passado ou mais recentemente, empregaram mais agressão psicológica, enquanto apenas uma cuidadora que sofrera episódios recentes de violência doméstica relatou comportamentos negligentes e fisicamente agressivos. Assim, na amostra pesquisada, ser vítima de violência conjugal estava associado a autorrelatos de maternagem agressiva e de disciplina negligente entre as cuidadores.

Nesse mesmo sentido, Renner (2009) examinou a relação entre violência conjugal e características da maternagem em amostra de 1,153 mulheres com baixa renda. Os resultados sugerem que sintomas depressivos medeiam a relação entre a vitimização psicológica da mãe e os autorrelatos de estresse parental.

Já Taylor et al.(2009) examinaram a associação 
entre a violência conjugal e os fatores de risco de a mãe vir a praticar maus-tratos contra a criança em amostra diversa de mães. Os dados do estudo foram obtidos a partir de amostra de 2.508 mães que participaram da pesquisa Famílias Frágeis e Bem-estar Infantil (Fragile Families and Child WellBeing Study). Os pesquisadores realizaram análise regressiva para examinar a associação entre violência conjugal, estresse parental, depressão maior e quatro variáveis para maus-tratos infantis maternos. Os resultados obtidos demonstraram que as mães relataram, em média, 25 atos de agressão psicológica e 17 atos de agressão física contra os filhos de 3 anos de idade no ano anterior ao estudo, $11 \%$ relataram algum ato de negligência em relação à criança durante o mesmo período e $55 \%$ agrediram fisicamente os filhos durante o mês anterior. Aproximadamente $40 \%$ das mães sofreram violência conjugal pelo parceiro atual. Tanto a violência contra a mulher quanto o estresse materno foram fatores de risco consistentes com as quatro variáveis de maus-tratos. Embora mães que tiveram os filhos em outros países tenham relatado menos incidentes de maus-tratos infantis, o risco relativo de violência contra a mulher para os maus-tratos infantis foi maior para essas mães do que para aquelas que tiveram os filhos nos Estados Unidos.

A fim de examinar a relação entre a experiência da mulher com violência conjugal e a qualidade da maternagem, Casanueva et al. (2008) utilizaram os dados da Pesquisa Nacional sobre o Bem-Estar da Criança e do Adolescente, em amostra de 1.943 mães de crianças com menos de 10 anos de idade investigadas por maus-tratos infantis. Os resultados indicaram que mulheres vítimas de violência conjugal no passado mas que, no momento da pesquisa, não eram vítimas, tinham escores de maternagem melhores quando comparadas com mulheres que estavam sendo vítimas de violência conjugal no presente, quando outros fatores de risco eram controlados. Esses dados demonstram que a violência conjugal não prejudica necessariamente a maternagem, o que indica a necessidade de uma avaliação caso a caso dos serviços de que a mulher necessita.

Em um estudo qualitativo, Damant et al. (2010) entrevistaram 27 mulheres vítimas de violência conjugal durante os 24 meses anteriores à coleta de dados que tinham pelo menos um filho com menos de 18 anos e que tinham acesso a serviço de saúde ou de assistência social para: (1) identificar as condições nas quais as mulheres desempenham a sua maternagem, (2) os efeitos que a violência conjugal tem nas habilidades maternas e como ela se relacionaria aos maus-tratos infantis, (3) explorar as experiências das mulheres com os serviços de saúde e de assistência social e (4) verificar como as mulheres veem a influência de sua maternagem na relação que estabelecem com os filhos e o seu contato com os serviços de saúde e de assistência social. De acordo com as pesquisadoras, a violência perpetrada pelas mulheres contra os filhos pode ser vista como uma consequência de sua própria vitimização, contudo, os autores consideram que as mulheres devem ser responsabilizadas pela escolha de utilizar violência contra as crianças.

Kelly (2009) realizou um estudo descritivo a respeito do processo de tomada de decisão de mães de origem hispânica que vivem nos EUA que sofriam violência conjugal. Os resultados obtidos demonstraram que o papel materno é central nas decisões dessas mulheres. Elas afirmaram lutar para priorizar, proteger e prover suas crianças de qualquer maneira, o que incluía manejar o abuso e evitar a revelação para profissionais de saúde. 
De modo geral, as pesquisas descritas acima demonstram que a violência conjugal tem impactos negativos nas habilidades maternas, aumentando a probabilidade de elas praticarem algum tipo de mau-trato infantil, sendo a agressão psicológica a mais frequente, seguida de agressão física e negligência. Vale destacar que o impacto é maior quanto mais próximo for o episódio de violência conjugal, sendo as consequências minimizadas com o passar do tempo. Tais dados indicam a importância de se considerar a avaliação da maternagem das mulheres quando a violência conjugal é revelada, como forma de prevenir maus-tratos infantis ou o agravamento de problemas na relação mãefilhos. Por outro lado, há que se ter em conta fatores de proteção como, por exemplo, a alta escolaridade, e, assim, lembrar que algumas mães com histórico de violência podem desempenhar sua maternagem melhor do que outras.

\section{Impacto da violência conjugal nas crianças}

Dos sete estudos a respeito do impacto que a violência conjugal ocasiona nas crianças, quatro identificaram problemas de comportamento internos e externos apresentados pelas crianças (GrahamBermann, Gruber, Howell, \& Girz, 2009; Zerk, Mertin, \& Proeve, 2009; Paterson, Carter, Gao, Cowley-Malcolm, \& Iusitini, 2008; Hughes \& Huth-Bocks, 2007), um estudo analisou as reações das crianças em situações com pares (Katz, Hunter, \& Klowden, 2008) e um estudo verificou o impacto na memória explícita de préescolares (Jouriles, Brown, McDonald, Rosenfield, Leahy, \& Silver, 2008).

Entre os estudos que analisaram o impacto da violência conjugal nas crianças, destaca- se o de Graham-Bermann, Gruber, Howell e Girz (2009), que avaliou o ajustamento emocional e social de crianças de famílias com graus variados de violência contra o parceiro utilizando o modelo de risco e proteção. Duzentas e dezenove mães que sofreram violência do parceiro no último ano e os filhos com idades entre 6 e 12 anos foram entrevistados. Medidas padronizadas avaliaram a violência familiar, a maternagem, o funcionamento familiar, a saúde mental da mãe e o ajustamento e as crenças das crianças. Os resultados das crianças foram divididos em quatro perfis: problemas severos de ajustamento (24\%), crianças que estavam tendo dificuldades (45\%), crianças que tinham exclusivamente depressão (11\%) e crianças resilientes (20\%), com alta competência e poucos problemas de ajustamento. Análise de regressão logística multinomial demonstrou que as crianças com problemas de ajustamento severos foram mais expostas à violência familiar e tinham mães com maior índice de depressão e sintomas de trauma do que as outras crianças. As crianças resilientes e do grupo que estava tendo dificuldades tinham mães com melhor maternagem, maior força familiar e nenhum histórico de violência conjugal anterior ao atual. As crianças depressivas estavam expostas a menos violência, mas tinham mais preocupação e medo pela segurança de suas mães. Os achados sugerem que o ajustamento da criança é fortemente influenciado pelo funcionamento parental.

O estudo de Finger et al. (2009) examinou a inter-relação entre conflito familiar, suporte do pai à mãe, comportamento materno e apego mãe-crianca de uma amostra de 79 famílias afro-americanas que viviam em uma comunidade urbana. O suporte do pai à mãe não foi relacionado à maternagem ou ao apego infantil. As relações conflituosas entre 
os pais foram relacionados à maternagem problemática, baixa sensitividade materna, apego inseguro da criança e apego desorganizado da criança. As associações entre conflito parental e apego inseguro e desorganizado foram minimizados em famílias nas quais os pais coabitavam. Consistente com pesquisas anteriores, foram observadas relações entre sensitividade e responsividade maternal e o apego seguro com os filhos e entre maternagem problemática e apego desorganizado da criança. Entretanto, a maternagem não mediou a relação entre conflito parental e apego das crianças.

Já Paterson et al. (2008) objetivaram examinar: (1) a associação entre ser vítima e agressor de violência conjugal e problemas de comportamento entre crianças das ilhas do Pacífico de 2-4 anos de idade e (2) fatores sociodemográficos e parentais que podem influenciar essa associação. Mães das famílias locais e crianças nascidas na Nova Zelândia no ano 2000 foram entrevistadas. Os dados foram coletados com mães que eram casadas e coabitavam com o parceiro $(N=920)$ e que completaram as medidas de violência conjugal nas duas avaliações realizadas e o Child Behaviour Checklist (CBCL). Dessas, 709 mães completaram o CBCL nos dois pontos de avaliação. Os resultados obtidos não demonstraram associações significativas entre a violência conjugal e a prevalência das taxas de casos clínicos relevantes de problemas comportamentais na amostra de crianças de dois anos. Entretanto, as taxas de prevalência de problemas internos, externos e totais foram significativamente maiores para as crianças de quatro anos de idade cujas mães relataram sofrer violência conjugal severa. A probabilidade de estar na faixa interna foi 2,16 vezes maior para os filhos de mães que os agrediam fisicamente do que para aqueles em que as mães não os agrediam, sendo que, para problemas externos e totais, a probabilidade foi, respectivamente, 2,38 e 2,36 vezes maior. Fatores sociodemograficos e parentais não influenciaram significativamente a associação entre violência conjugal e problemas de comportamento da criança.

Hughes e Huth-Bocks (2007) verificaram os efeitos dos fatores contextuais (estresse parental, comportamentos parentais e funcionamento psicológico da mãe) em crianças expostas à violência conjugal. Participaram da pesquisa 172 mães afroamericanas e seus filhos (4 a 12 anos de idade) que estavam em um abrigo no momento da pesquisa. As mães preencheram questionários que avaliavam a violência familiar, as variáveis contextuais da família e as consequências nos filhos. Já as crianças responderam sobre seus sintomas depressivos. Os resultados indicaram uma variabilidade substancial entre as experiências de estresse parental, tanto em relação ao tipo quanto à quantidade. As mulheres diferiram significativamente nos comportamentos maternos e no estresse psicológico geral, e as crianças variaram de forma simultânea na severidade dos problemas internos e externos. Esse padrão sugere a necessidade de intervenções individuais focadas no estresse parental para melhor atender as necessidades de mulheres e crianças vítimas de violência conjugal.

No estudo de Zerk, Mertin e Proeve (2009), 60 mães foram entrevistadas para investigar as respostas de trauma de crianças em idade pré-escolar que viviam em lares nos quais a violência conjugal estava presente. Os sintomas de trauma foram mensurados utilizando-se adaptações de critérios de desenvolvimento baseados no Child 
Behavior Checklist (CBCL), e dados da mãe também foram avaliados quanto à ansiedade, depressão, somatização e estresse parental. Os resultados obtidos sugerem que, além dos níveis clínicos de problemas internos, externos e totais do $\mathrm{CBCL}$, as crianças menores apresentam uma gama de sintomas pós-traumáticos. Mães que relataram um alto nível de violência e um relacionamento significativo apresentaram níveis de angústia e estresse parental, sendo este último o preditor mais forte dos escores do CBCL. Embora a violência doméstica sozinha não tenha apresentado uma correlação significativa com os resultados das crianças, os dados indicam que a angústia materna tem um impacto adverso na relação mãe-filho.

Buscando relacionar a exposição à violência conjugal e as reações da criança nas interações com os pares em amostra baseada na comunidade, Katz, Hunter e Klowden (2008) convidaram crianças para responder a um modelo de provocação de pares, e as mães das mesmas completaram uma entrevista metaemocional parental (Parent Meta-Emotion Interview) (Gottman, Katz, \& Hooven, 1997). Reações adequadas (rir, ignorar) e não adequadas (hostil, desafiador, comportamentos estranhos) para as provocações dos pares foram examinadas. Os resultados indicaram uma relação positiva entre violência conjugal e comportamentos estranhos e comportamentos de rir. Além disso, o manejo de emoções moderou a relação entre violência conjugal e os comportamentos estranhos e de dar risada das crianças.

Jouriles et al. (2008) verificaram se a violência física conjugal se relaciona com o funcionamento de memória explícita de préescolares, se os sintomas de hiperatividade mediavam essa relação e se a maternagem positiva moderava a mesma. Participaram do estudo 69 mães e seus filhos de 4-5 anos de idade (34 meninas). As mães completaram medidas de violência conjugal, sintomas de hiperatividade infantil, agressão mãecriança e maternagem positiva. Medidas de memória explícita foram administradas aos pré-escolares. Como esperado, a violência conjugal teve uma correlação negativa com desempenho dos pré-escolares nas tarefas de memória explícita, mesmo quando se controlaram algumas variáveis como agressão pais-criança e variáveis demográficas associadas ao funcionamento da memória de pré-escolares. Os sintomas de hiperatividade dos pré-escolares não mediaram a relação entre violência conjugal e funcionamento da memória explícita, mas a maternagem positiva moderou essa relação, isto é, a relação entre violência conjugal e os resultados negativos das crianças entre duas ou três tarefas de memória explícita foram menores quando as mães se engajaram em níveis maiores de maternagem positiva.

As pesquisas descritas acima demonstram que estar exposto à violência conjugal leva a problemas de comportamentos internos e externos nas crianças, sintomas póstraumáticos, assim como a problemas no relacionamento com pares e em atividades de memória explícita em crianças préescolares. Dentre os fatores moderadores desses sintomas, destacam-se a qualidade da maternagem e a gravidade da violência conjugal ocorrida. Mais uma vez, o conjunto de pesquisas analisadas ressalta a importância de intervir com as mulheres vítimas de violência conjugal no sentido de as mesmas lidarem com os sintomas da agressão e de conseguirem minimizar os seus efeitos no relacionamento com os filhos. 
Cabe destacar, contudo, que, dentre os estudos que verificaram os impactos da violência conjugal nas habilidades maternas,

apenas alguns utilizaram gruposcontrole (Duggan et al., 2009; Jouriles et al., 2009).

\section{Considerações finais}

Os artigos analisados demonstram que uma parcela considerável de crianças norteamericanas presencia desentendimentos violentos em seus lares (Moore et al., 2007) e que grande parte dos casos de violência doméstica investigados pelo sistema judiciário norte-americano foi testemunhado por crianças (Fusco \& Fantuzzo, 2009), o que indica a necessidade de se ter uma avaliação global de todos os membros da família para se ponderar as consequências da violência ocorrida, visto que a criança exposta a violência pode apresentar diversas sequelas no seu desenvolvimento cognitivo e emocional (Holt, Buckley, \& Whelan, 2008; Brancalhone \& Williams, 2003; Azevedo \& Guerra, 1995; Sinclair, 1985).

Nesse sentido, alguns dos estudos analisados demonstraram os impactos que a violência conjugal tem no surgimento de problemas de comportamento das crianças (GrahamBermann et al., 2009; Zerk et al., 2009; Paterson et al., 2008; Jouriles et al., 2008; Katz et al., 2008; Hughes \& Huth-Bocks, 2007). Cabe destacar que, mesmo quando não foi encontrada uma correlação positiva entre a violência conjugal e os problemas de comportamento da criança (Zerk et al., 2009), sintomas decorrentes da violência sofrida pela mulher, como angústia e depressão, por exemplo, foram correlacionadas a dificuldades no manejo comportamental e emocional das crianças.

Além disso, todos os estudos indicaram que os comportamentos maternos mais adequados eram um preditor maior de ajustamento das crianças, o que tem implicações práticas e sugere que uma intervenção efetiva seria aquela em que mulheres vítimas de violência doméstica recebessem apoio, "tanto para a questão da violência", quanto para a educação dos filhos, como pode ser observado nos dados obtidos por Jouriles et al. (2009) e Casanueva et al. (2008). Vale destacar que o LAPREV (Laboratório de Análise e Prevenção da Violência) é pioneiro nessa área no Brasil, tendo implantado, em 2008, o Projeto Parceria, que oferece atendimento psicológico a mães vítimas de violência doméstica mesclando componentes psicoterapêuticos e educacionais, e tem obtido dados positivos no sentido de minimizar as dificuldades no manejo do comportamento dos filhos e de melhorar o relacionamento das mães com os filhos, capacitando-as para lidar com a situação vivenciada (Williams, Padovani, \& Brino, 2009; Williams, Araújo, Rios, D'Affonseca, Maldonado, Patrian, \& Miranda, 2010).

Em relação às habilidades maternas, a maioria dos estudos analisados demonstrou impactos negativos da exposição à violência conjugal, com um aumento do estresse parental das mães (Renner, 2009) e de comportamentos agressivos por parte da mãe (Damant et al., 2010; Taylor et al., 2009; Kelleher et al., 2008), sendo que Letourneau, Fedick e Willms (2007) demonstraram que mães de crianças expostas à violência doméstica podem, de fato, compensar a exposição à violência dos filhos nas suas interações maternas, dando indicativos de que algumas mulheres conseguem superar sua experiência de vitimização e oferecer maternagem adequada aos filhos. Cabe destacar, contudo, que, dentre os estudos que verificaram os impactos da violência conjugal nas habilidades maternas, apenas alguns utilizaram gruposcontrole (Duggan et al., 2009; Jouriles et al., 2009). Adicionalmente, todos os estudos analisados contaram exclusivamente com o autorrelato das mães como fonte de informação. Não foram encontrados estudos 
que verificassem as habilidades maternas por meio de outras fontes, como, por exemplo, a observação da interação dos mesmos; assim, seria importante que estudos observacionais sistematizados fossem realizados com essa população para que se pudesse verificar o repertório dessas mulheres.

\section{Sabrina Mazo D'Affonseca}

Doutoranda do Programa de Pós-Graduação em Psicologia da Universidade Federal de São Carlos, São Carlos - SP - Brasil.

E-mail: samazo@hotmail.com

\section{Lúcia Cavalcanti de Albuquerque Williams}

Professora Titular do Departamento de Psicologia da Universidade Federal de São Carlos,

São Paulo - SP - Brasil.

E-mail:williams@power.ufscar.br

Endereço para envio de correspondência:

LAPREV - Laboratório de Análise e Prevenção da Violência, Universidade Federal de São Carlos,

Departamento de Psicologia Caixa Postal 676, São Carlos - SP - Brasil. CEP: 13.565-905 


\section{Referências}

Adeodato, V. G., Carvalho, R. R., Siqueira, V. R., \& Souza, F. G. M. (2005). Qualidade de vida e depressão em mulheres vítimas de seus parceiros. Revista de Saúde Pública, 39(1), 108-113.

Appel, A. E., \& Holden, G. W. (1998). Co-occurring spouse and child abuse: Implications for CPS practice. APSAC Advisor, 11(1), $11-14$.

Arias, I., \& Ikeda, R. M. (2006). Etiology and surveillance of intimate partner violence. In J. R. Lutzker (Ed.), Preventing violence: Research and evidence-based intervention strategies. (pp.173-194). Washington, DC: American Psychological Association.

Azevedo, M. A. \& Guerra, V.N.A. (1995) Infância e violência fatal em família. São Paulo: Iglu Editora.

Brancalhone, P.G. \& Williams, L.C.A. (2003). Crianças expostas à violência conjugal: Uma revisão de área. In M.C. Marquezine, M.A. Almeida, S. Omote e E.D.O. Tanaka (Orgs.). O papel da família junto ao portador de necessidades especiais. (pp. 123130, Coleção Perspectivas Multidisciplinares em Educação Especial, 6). Londrina, PR: Eduel.

Capaldi, D. M., Kim, H., \& Pears, K. (2009). The association between partner violence and child maltreatment: A common conceptual framework. In D. J. Whitaker \& J. R. Lutzker, Preventing partner violence: Research and evidencebased intervention strategies (pp. 93-111). Washington, DC: American Psychological Association.

Casanueva, C., Martin, S. L., \& Runyan, D. K. (2009). Repeated reports for child maltreatment among intimate partner violence victims: Findings from the national survey of child and adolescent well-being. Child Abuse and Neglect, 33, 84-93.

Casanueva, C., Martin, S. L., Runyan, D. K., Barth, R. P., \& Bradley, R. H. (2008). Parenting services for mothers involved with child protective services: Do they change maternal parenting and spanking behaviors with young children? Children and Youth Services Review, 30(8), 861-878.

Casanueva, C., Martin, S. L., Runyan, D. K., Barth, R. P., \& Bradley, R. H. (2008). Quality of maternal parenting among intimate-partner violence victims involved with the child welfare system. Journal of Family Violence, 23, 413-427.

Chaffin, M., \& Schmidt, S. (2006). An evidence-based perspective on interventions to stop and prevent child abuse. In J. R.
Lutzker (Ed.), Preventing violence: Research and evidence-based intervention strategies (pp.49-68). Washington, DC: American Psychological Association.

Constantino, R., Crane, P. A., Noll, B. S., Doswell, W. M., \& Braxter, B. (2007). Exploring the feasibility of email-mediated interaction in survivors of abuse. Journal of Psychiatric \& Mental Health Nursing, 14(3), 291-301.

Dahlberg, L. L., \& Simon, T. R. (2006). Predicting and preventing youth violence: Developmental pathways and risk. In J. R. Lutzker (Ed.), Preventing violence: Research and evidence-based intervention strategies (pp.97-124). Washington, DC: American Psychological Association.

Damant, D., Lapierre, S., Lebosse, C., Thibault, S., Lessard, G., Hamelin-Brabant, L., Lavergne, C., \& Fortin, A. (2010). Women's abuse of their children in the context on domestic violence: Reflection from women's accounts. Child and Family Social Work, 15, 12-21.

Duggan, A. K., Berlin, L. J., Cassidy, J., Burrell, L., \& Tandon, S. D. (2009). Examining maternal depression and attachment insecurity as moderators of the impacts of home visiting for at-risk mothers and infants. Journal of Consulting and Clinical Psychology, 77(4), 788-99.

Edelson, M. G., Hokoda, A., \& Ramos-Lira, L. (2007). Differences in effects of domestic violence between Latina and non-Latina women. Journal of Family Violence, 22(1), 1-10.

Finger, B., Hans, S. L., Bernstein, V. J., \& Cox, S. M.Parent relationship quality and infant-mother attachment. Attachment and human development, 11(3), 285-306.

Fusco, R. A., \& Fantuzzo, J. W. (2009). Domestic violence crimes and children: A population-based investigation of direct sensory exposure and the nature of involvement. Children \& Family Services Review, 31, 249-256.

Graham-Bermann, S. A., Gruber, G., Howell, K. H., \& Girz, L. (2009). Factors discriminating among profiles of resilience and psychopathology in children exposed to intimate partner violence (IPV). Child Abuse and Neglect, 33(9), 648-60.

Gottman, J. M., Katz, L. F., \& Hooven, C. (1997). Meta-emotion: How families communicate emotionally. Hillsdale, NJ: Lawrence Erlbaum. 
Hermann, J., \& Barsted, L. L. (2000). Violência contra a mulher: um guia de defesa, orientação e apoio. Rio de Janeiro: CEPIA/ CEDIM.

Holden, G. W. (2007, maio). Abused women as mothers: Evidence, issues and controversies. In Third International Conference on Children Exposed to Family Violence, London, Canada,

Holigrocki, R., Crain, R., Bohr, Y., Young, K., \& Bensman, H. (2009). Interventional use of the parent-child interaction assessment-II enactments: Modifying an abused mother's attributions to her son. Journal of Personality Assessment, 91(5), 1-12.

Holt, S., Buckley, T., \& Whelan, S. (2008). The impact of exposure to domestic violence on children and young people: A review of the literature. Child Abuse \& Neglect, 32(8), 797-810.

Hughes, H.M. \& Huth-Bocks, A.C. (2007). Variations in parenting stress in African-American battered women: Implications for children's adjustment and family intervention. European Psychologist, 12, 62-71.

Jouriles, E. N., McDonald, R., Rosenfield, D., Stephens, N., Corbitt-Shindler, D., \& Miller, P. C. (2009). Reducing conduct problems among children exposed to intimate partner violence: A randomized clinical trial examining effects of project support. Journal of Consulting and Clinical Psychology, 77(4), 705-17.

Jouriles, E. N., Brown, A. S., McDonald, R., Rosenfield, D., Leahy, M. M., \& Silver, C. (2008). Intimate partner violence and preschoolers' explicit memory functioning. Journal of Family Psychology, 22, 420-428.

Katz, L. F., Hunter, E., \& Klowden, A. (2008). Intimate partner violence and children's reaction to peer provocation: The moderating role of emotion coaching. Journal of Family Psychology, 22(4), 614-621.

Kelleher, K. J., Hazen, A. L., Coben, J. H., Wang, Y., McGeehan, J., Kohl, P. L., \& Gardner, W. P. (2008). Self-reported disciplinary practices among women in the child welfare system: Association with domestic violence victimization. Child Abuse and Neglect, 32(8), 811-8.

Kelly, U. A. (2009). "I'm a mother first": The influence of mothering in the decision-making processes of battered immigrant latino women. Research in Nursing \& Health, 32(3), 286-97.

Lutzker, J. R. (2006). Preventing violence: Research and evidencebased intervention strategies. Washington, DC: American Psychological Association.

Mele, M. (2009). The time course of repeat intimate partner violence. Journal of Family Violence, 24(8), 619-624.

Moore, C. G., Probst, J. C., Tompkins, M., Cuffe, S., Martin, A. B. (2007). The prevalence of violent disagreements in US families: Effects of residence, race/ethnicity, and parental stress. Pediatrics, 119(1), S68-76.

O'Leary, K. D., \& Woodin, E. M. (2006). Bringing the agendas together: Partner and child abuse. In J. R. Lutzker (Ed.), Preventing violence: Research and evidence-based intervention strategies (pp. 239-258). Washington, DC: American Psychological Association.

Paterson, J., Carter, S., Gao, W., Cowley-Malcolm, E., \& lusitini, L. (2008). Maternal intimate partner violence and behavioural problems among Pacific children living in New Zealand. Journal Child Psychology Psychiatry, 49(4), 395-404.

Probst, J. C., Wang, J. Y., Martin, A. B., Moore, C. G., Paul, B. M., \& Samuels, M. (2008). Potentially violent disagreements and parenting stress among American Indian/Alaska Native families: Analysis across seven states. Maternal and Child Health Journal, 12(1), 91-102.

Renner, L. M. (2009). Intimate partner violence victimization and parenting stress: Assessing the mediating role of depressive symptoms. Violence Against Women, 15(11), 1380-1401.

Rios, K., D'Affonseca, S. M., Santini, P. M., Lessa, T., Patrian, A. A., Miranda, T. D. \& Williams, L. C. A. (2010). Uma casa-laboratório como recurso tecnológico para o ensino de habilidades parentais e prevenção de problemas do comportamento infantil. In Sobre comportamento e cogniçã: Terapia comportamental e cognitivas (Vol. 27, pp. 210-222). Santo André, SP: ESETec.

Sinclair, D. (1985). Understanding wife assault. Toronto: Publications Ontario.

Slep, A. M. S., \& O'Leary, S. G. (2001). Examining partner and child abuse: Are we ready for an integrated theory of family violence? Child and Family Psychology Review, 4, 87-108.

Straus, M. A., Hamby, S. L., Boney-McCoy, S., \& Sugarman, D. B. (1996). The revised conflict tactics scales (CTS2): Development and preliminary psychometric data. Journal of Family Issues, 17(3), 283-316.

Taylor, C. A., Guterman, N. B., Lee, S. J., \& Rathouz, P. J. (2009). Intimate partner violence, maternal stress, nativity, and risk for maternal maltreatment of young children. American Journal of Public Health, 99(1), 175-83.

Tischler, V., Rademeyer, A., \& Vostanis, P. (2007). Mothers experiencing homelessness: Mental health, support and social care needs. Health and Social Care in the Community, 15(3), 246-253.

Vasconcelos, I. F. (2002). Resolução violenta de conflitos entre casais: quem sai perdendo? In 13 Encontro da Associação Brasileira de Estudos Populacionais. Ouro Preto, MG.

Williams, L. C. A. (2001). Violência doméstica: há o que fazer? In H. J. Guilhardi, M. B. B. Madi, P. P. Queiroz, \& M. C. Scoz (Orgs.), Sobre o comportamento e cognição: expondo a variabilidade (Vol. 7, pp.1-12). Santo André, SP: ESETec.

Williams, L. C. A., \& Araujo, E. A. (2009). Habilidades maternas de mulheres que sofrem violência do parceiro: uma revisão. In R.C. Wielenska (Org.), Sobre comportamento e cognição: Desafios, soluções e questionamentos. (pp. 96-109). Santo André, SP: ESETec.

Williams, L. C. A., Padovani, R. C., \& Brino, R. F. (2009). O empoderamento da família para enfrentar a violência doméstica. São Carlos, SP: EDUFSCar/OPAS.

Williams, L. C. A., Araújo, E. A. C., Rios, K. A., D’Affonseca, S. M., Maldonado, D. A., Patrian, A. C. A., \& Miranda, T. D. (2010). Projeto Parceria: o ensino de habilidades parentais a mães com histórico de violência conjugal. In L. C. A. Williams, J. M. Maia, \& K. A. Rios. Aspectos psicológicos da violência: pesquisa e intervenção. (pp.428-457). Santo André, SP: ESETec.

Zerk, D. M., Mertin, P. G., \& Proeve, M. (2009). Domestic violence and maternal reports of young children's functioning. Journal of Family Violence, 24(7), 423-432. 